\title{
SCANNING TUNNELING MICROSCOPY OF PLATINUM ELECTRODE SURFACES WITH DIFFERENT PREFERRED CRYSTALLOGRAPHIC ORIENTATIONS
}

\author{
L. VÁZQUEZ, J.M. GÓMEZ RODRÍGUEZ, J. GÓMEZ HERRERO, \\ A.M. BARÓ, N. GARCÍA \\ Departamento de Fisica Fundamental, Universidad Autónoma de Madrid, Cantoblanco, \\ 28049 Madrid, Spain
}

\section{J.C. CANULLO and A.J. ARVÍA * \\ Instituto de Investigaciones Fisicoquímicas Teóricas y Aplicadas, Universidad Nacional de La Plata, Sucursal 4, Casilla de Correo 16, 1900 La Plata, Argentina}

Received 1 August 1986: accepted for publication 15 September 1986

We have used scanning tunneling microscopy to study the surface microtopography of platinum electrodes preferentially oriented by a procedure denoted as electrochemical faceting. The treated specimens show clear reoriented patches on the surface in the form of steps with well-defined orientations separated by terraces of different sizes. The microscopic data obtained by STM correspond well with voltammetric analysis.

\section{Introduction}

Preferred crystallographic orientations of polycrystalline platinum electrodes can be electrochemically obtained by the application of a relativcly fast symmetric periodic signal in the order of a few $\mathrm{kHz}$, between two potential limits lying within the range of the thermodynamic stability of bulk water $[1,2]$. This technique has been successfully applied to develop (100), (110) and (111)-type preferred orientations starting from either polycrystalline or single crystal specimens [3]. The development of different surface structures can be followed through the characteristics of the $\mathrm{H}$-adatom electroadsorption-electrodesorption spectra and their direct comparison to those reported in the literature for the corresponding single crystal platinum electrodes.

The new surface structure resulting after prolonged treatment $[1,2]$ can be observed by scanning electron microscopy (SEM) at a relatively low magnification $(\times 2000)$. Typical faceting patterns are observed for the different crystallites according to their direction in space [3-5].

\footnotetext{
* Visiting professor from the Universidad Nacional de La Plata, Argentina.
} 
The topography of the preferred oriented platinum surfaces can be studied with higher resolution by the application of scanning tunneling microscopy (STM). Recently we reported for the first time on the use of STM to measure the surface topography of preferred oriented platinum electrodes on the nanometer scale [6]. An STM study of electrochemically activated Pt electrodes has also been performed [7].

This paper presents a comparative study of (110), (100) and (111) preferred oriented platinum as measured by STM. In spite of big inhomogeneities in the data, we have found topographic features characteristic of well-defined oriented faces. The oriented patches have different sizes but can be as large as $1 \mu \mathrm{m} \times 1 \mu \mathrm{m}$. The microscopic observation of these structures gives some insight into the development of stable surface structures, and about the electrodissolution-electrodeposition process and represents a first attempt to correlate the macroscopic electrochemical data with microscopic observations by STM.

\section{Experimental}

Polycrystalline platinum specimens with preferred orientation in the (110), (100) and (111) directions were prepared as described elsewhere [1-3]. Their electrochemical characteristics were checked before and after the STM observations by following voltammetrically the response of the $\mathrm{H}$-adatom electroadsorption-electrodesorption reactions in $1 \mathrm{M} \mathrm{H}_{2} \mathrm{SO}_{4}$ at $25^{\circ} \mathrm{C}$. No voltammetric changes were observed either due to the storage period or because of the STM operation.

Untreated polycrystalline platinum electrode surfaces were taken out of the same wire or plate to be used as a blank experiment, after they had been mechanically polished to a mirror surface with the finest grade alumina powder. STM images were obtained at room temperature and $10^{-6}$ Torr with a third generation STM instrument [8]. It consists of a tunneling unit suspended by springs and provided with viton and eddy current dampers. The data were taken at a tunneling voltage of $-10 \mathrm{mV}$ (tip negative) and a tunneling current of $5 \mathrm{nA}$. A typical scanning frequency was $0.1 \mathrm{~Hz}$.

\section{Results}

Due to the large inhomogeneities observed on the electrodes we recorded wide scans in the order of $1 \mu \mathrm{m} \times 1 \mu \mathrm{m}$ to get a representative sampling of the electrode surface. As a general result, the electrofaceting treatment produces a surface topography smoother than that of the blank (fig. 1). In addition some of the regions show features characteristic of well-defined orientations. In the 


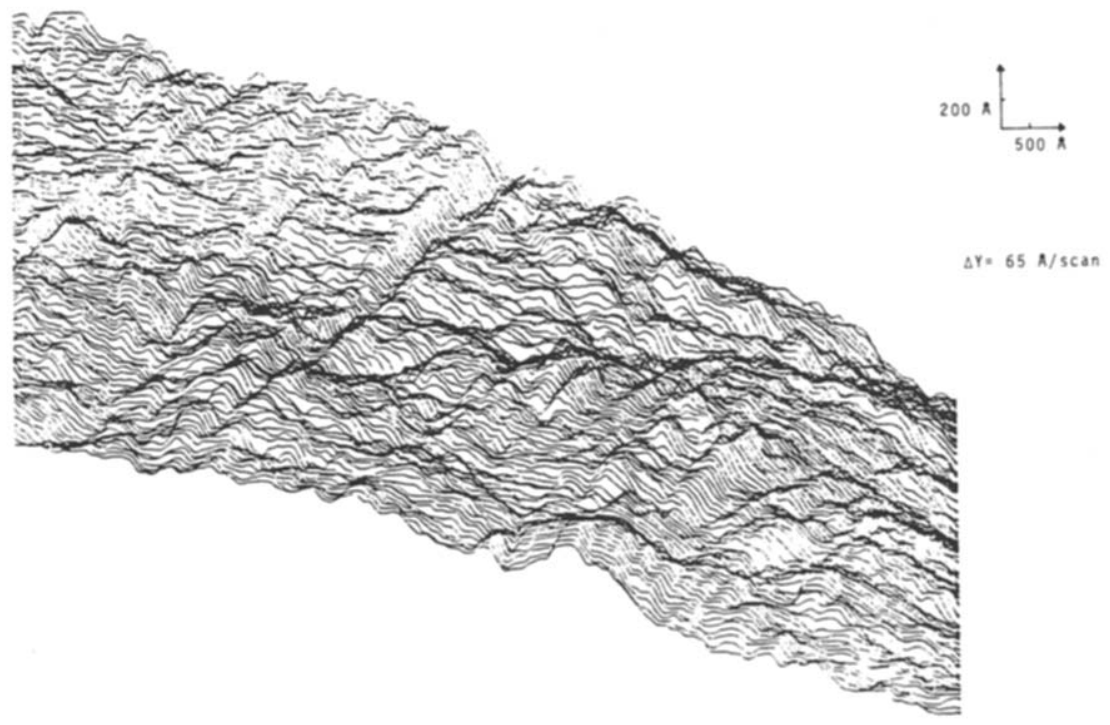

Fig. 1. STM image corresponding to the untreated polycrystalline (pc) Pt electrode showing a very corrugated surface.
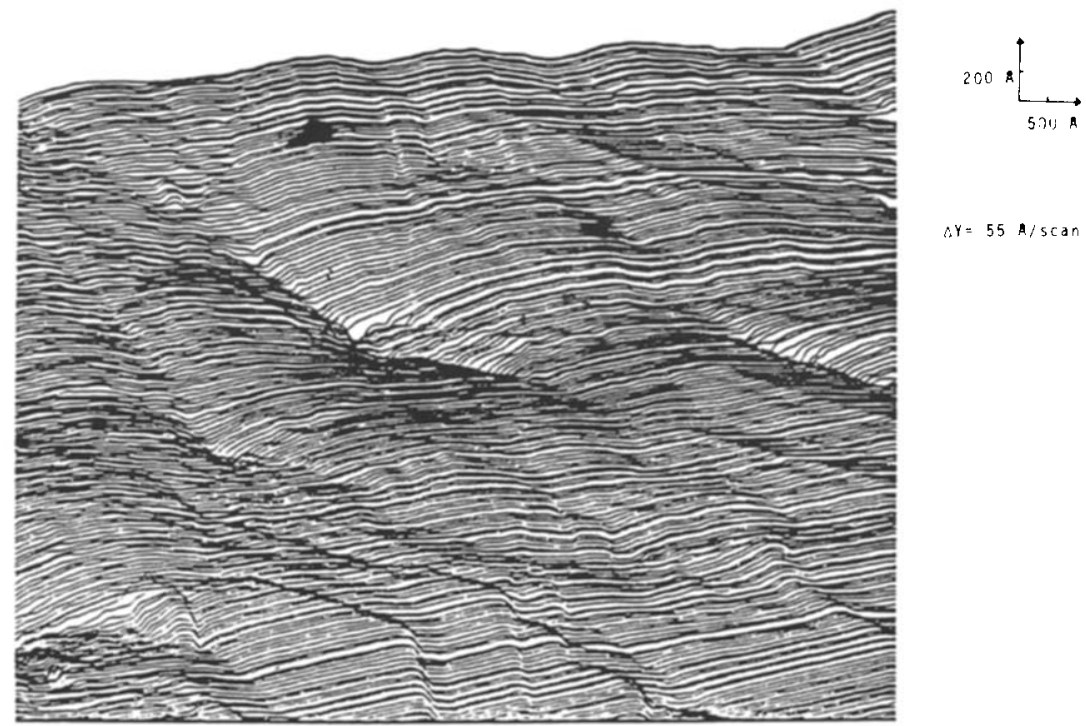

$\therefore Y=55 \mathrm{~A} / \mathrm{scan}$

Fig. 2. STM pattern of a (110)-type faceted pe Pt electrode. The picture shows (i) steps as deep as $100 \AA$ oriented in two different directions, (ii) flat regions with a wavy structure due to the development of steps which show up as parallel ridges, (iii) two wells separating the different flat regions 
following we report the features for the (110), (100) and (111) orientations.

The STM image of the (110) preferred oriented platinum (fig. 2 ) shows very well-defined steps as high as about $100 \AA$. Each step separates wide flat terraces extending up to $0.2-0.3 \mu \mathrm{m}$. The orientation of the steps is along two different directions that form an angle of approximately $120^{\circ}$. Furthermore, other flat regions can be observed showing a wave shaped structure with a corrugation of about $20-50 \AA$. These structures are related to the development of steps which show up as parallel ridges in the image. In the middle of the image two similar types of irregularities can be noticed consisting of abrupt walls forming a sort of well of at least about $100 \AA$ depth. Probably this type
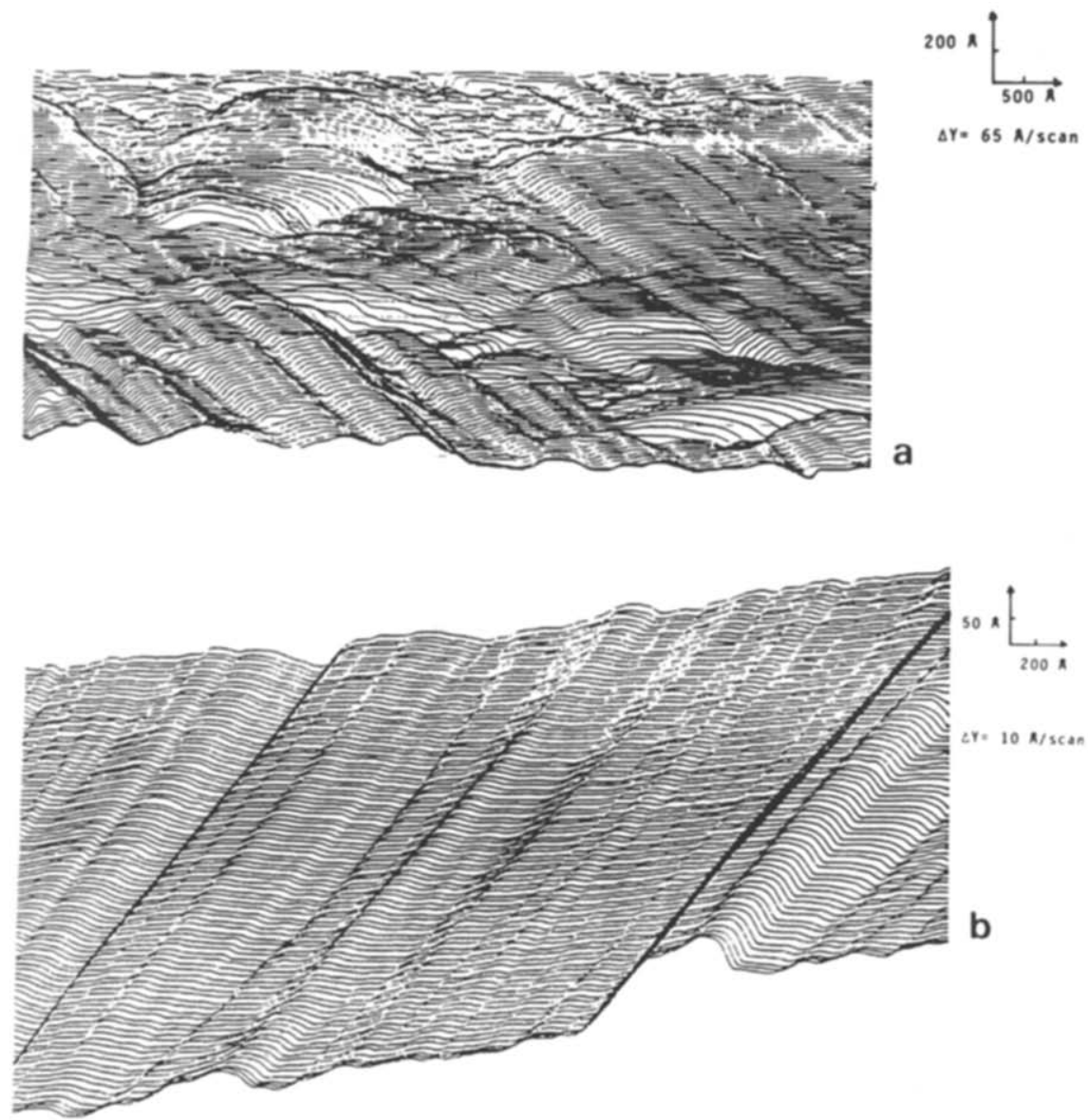

Fig. 3. (a) STM pattern of a (100)-type faceted pc Pt electrode. The picture shows the characteristic ridges corresponding to the development of steps. The picture shows that the oriented patches are quite small in size (submicrometer). The boundaries show up as grooves and wells. (b) One of the patches taken at higher magnification. 

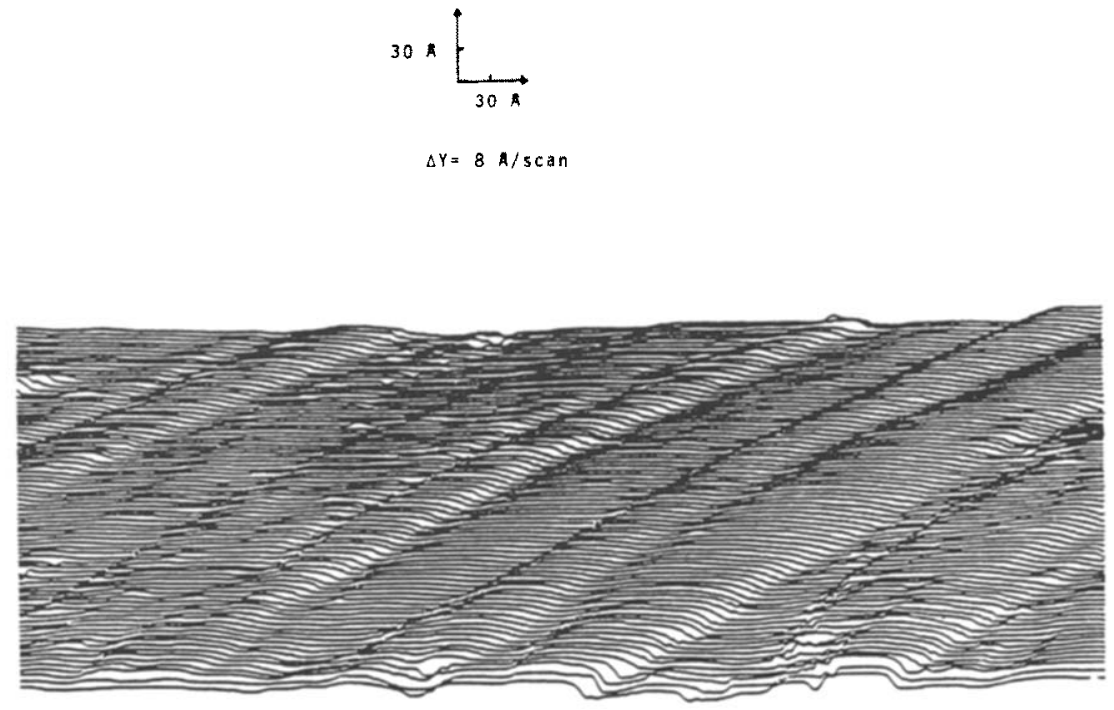

Fig. 4. STM pattern of a Pt(100) single crystal.

of accidents which are often seen in treated specimens reflect the remaining influence of the grain boundaries of the underlying bulk structure.

The images of the (100)-type preferred oriented platinum surfaces show very clear domains of crystallographic orientations as described in detail in a recent publication [6]. Typical examples of the corresponding topographies are depicted for low (fig. 3a) and high (fig. 3b) resolution, respectively. The structure of this orientation is to a large extent comparable to that already described for the (110)-type preferred oriented platinum. For the purpose of comparison we also measured the surface topography of a $\mathrm{Pt}(100)$ bulk single crystal in order to show the strong similarity with the electrochemically faceted specimen (fig. 4). The bulk single crystal had been previously used in surface science experiments in UHV and subsequently stored in the air atmosphere.

The STM images of (111)-type preferred oriented platinum exhibit a structure rather more complex that the one previously described. In principle the surface is constituted of two distinguishable regions. One of them is similar to that already reported for the (110) and (100) preferred crystallographic orientations, although in the present case it shows at least three definite directions (fig. 5a), the corresponding angles being consistent with the (111) trigonal symmetry. These patches, as compared to those observed for the (110) and (100)-type preferred orientations are much smaller in size. Another region is made out of a set of peaks of triangular shape which presumably correspond 

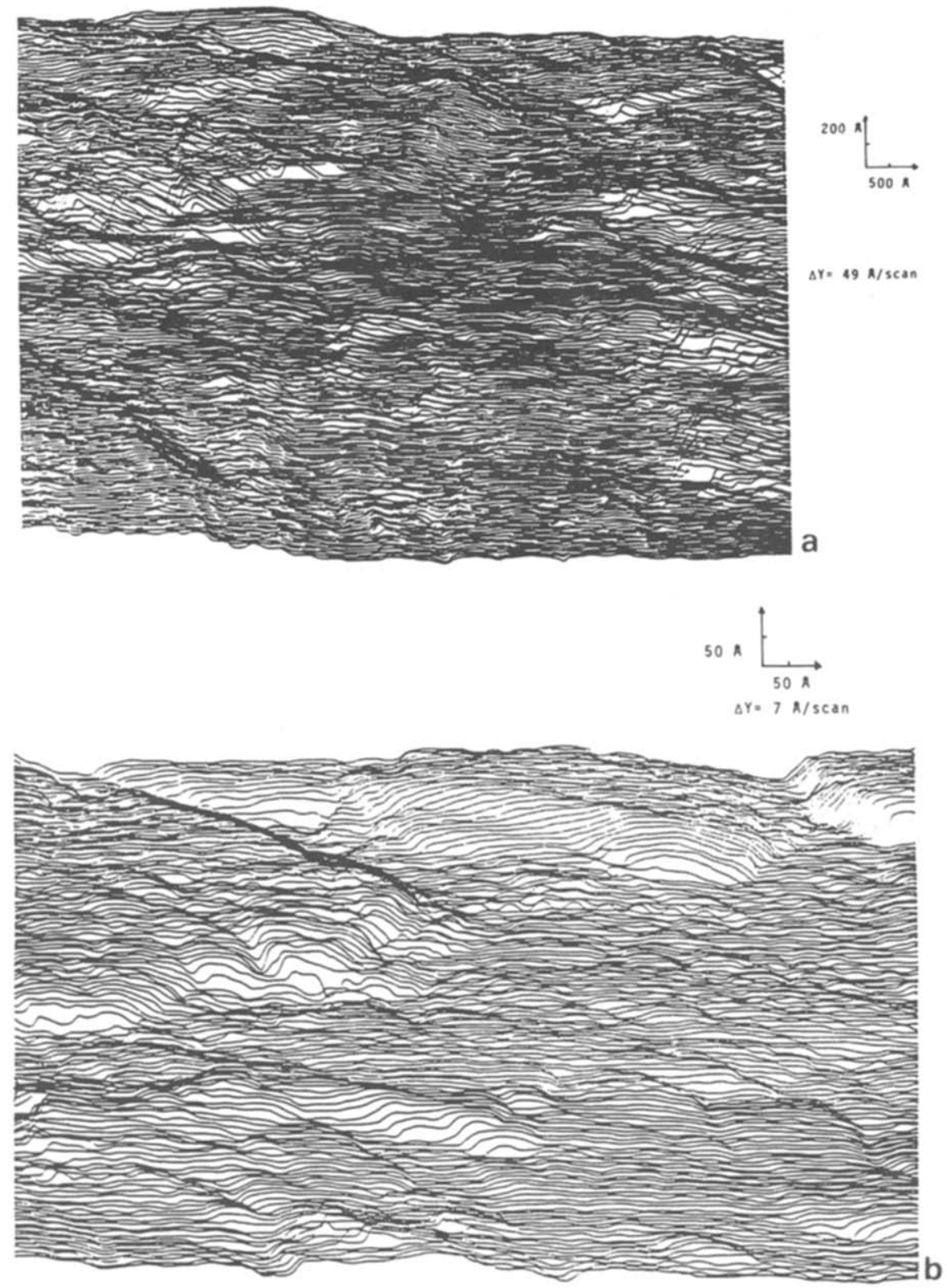

Fig. 5. (a) STM pattern of a (111)-type faceted pc Pt electrode. In addition to the ridges similar to those of $(110)$ and $(100)$-type faceted pc $\mathrm{Pt}$, structures in the form of sharp triangles are developed, probably related with the formation of $3 \mathrm{D}$ crystallites. (b) One of the patches taken at higher magnification. 
to three-dimensional polyhedrical crystallites of different heights ranging from 50 to $400 \AA$. In this case due to the abrupt changes produced by these structures the image could be distorted by the proper tip shape and/or time constant of the feedback response.

A higher resolution STM image (fig. 5b) shows a finer structure of the (111) preferred orientation. The image shows well-defined steps $50 \AA$ deep, oriented in three different directions. These steps constitute boundaries among different flat terraces. Each terrace shows a structure similar to that described before, i.e. well-defined orientations and triangular structures.

\section{Discussion}

STM provides the topographic images of small flat surface regions on the nanometer scale which usually cannot be observed by other techniques. STM images reveal that electrochemical faceting produces, in general, very smooth surfaces. Some of the regions show features characteristic of well-defined orientations such as aligned steps of a few atomic diameters height separated by flat terraces of different sizes. Other regions show deep valleys separated by rounded top hills. This topography presumably reflects the underlying bulk structure of the starting material. The STM topographies resulting for the three types of preferred oriented platinum surfaces present their peculiarities which in a much lower scale correlate with the corresponding SEM micrographs reported previously [3-5]. This is the case, for instance for the stepped surfaces observed for (110) preferred oriented platinum, the predominant parallel ridge structure observed for (100) preferred oriented specimens and the spike-like structure which appears typical for the (111)-preferred orientation.

Electrochemical faceting produced through fast periodic perturbation treatment covering potential limits no greater than those associated with the thermodynamic stability of bulk water, implies at least two concurrent electrochemical processes, namely the electroadsorption/electrodesorption of $\mathrm{OH}$ species and the electrodissolution/electrodeposition of soluble platinum species [9]. The occurrence of these two reactions under optimal conditions for electrochemical faceting are given by the frequency value of the periodic potential which must be at least in the order of a few $\mathrm{kHz}$. In this case the fast cyclic $\mathrm{OH}$-species electroadsorption/electrodesorption is responsible for the initial steps of platinum electrodissolution, so that the cyclic electrodissolution/electrodeposition of soluble platinum species can be regarded as a process occurring within a pulsating diffusion layer whose thickness decreases according to the frequency of the cyclic potential [10]. Therefore, under these circumstances one should expect the diffusional processes to occur very fast so that the rate limiting process is the electrodissolution/electrodeposition of 


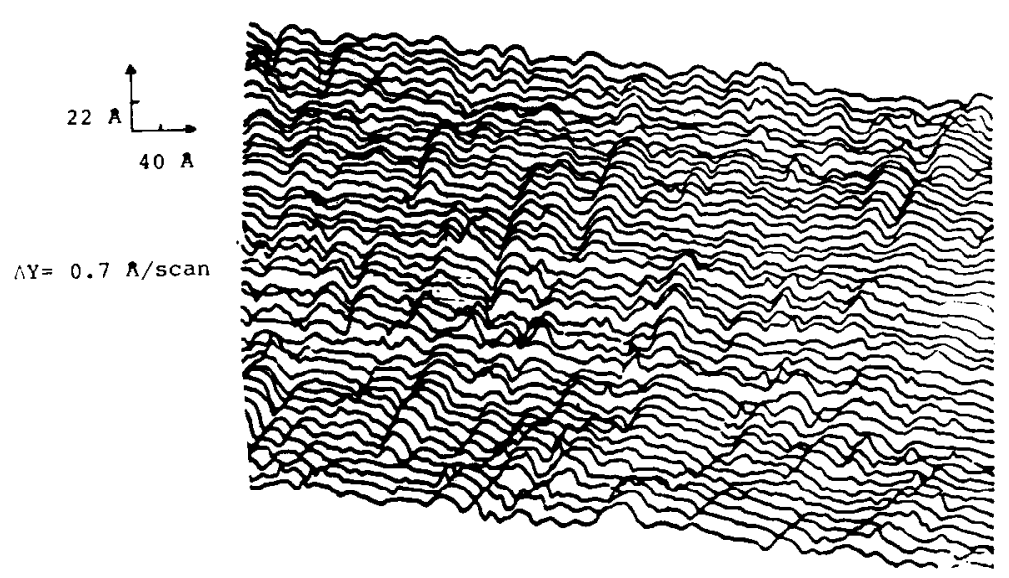

Fig. 6. STM image of a $50 \AA$ thick platinum film deposited on cleaved mica at room temperature.

soluble platinum. This process is sensitive to the reacting characteristics of the different sites of the metal surface. Therefore, the electrodeposition turns out to be a very selective process.

The different behaviour of the cyclic deposition process under electrochemical faceting conditions can be well understood by comparing the electrode surface with that obtained by a $50 \AA$ thick platinum film vacuum deposited on top of a freshly cleaved mica surface at room temperature (fig. 6). The image shows the so-called columnar structure which is characteristic of this metal growing condition [11], namely the substrate temperature is well below the melting temperature of the depositing material. Columns are formed around initial nuclei because of the small diffusion rate of the deposited atoms which does not allow covering of the intercolumnar regions. This is in contrast with the electrochemical treatment explained before, where due to the high frequency potential sweep, the process is controlled by the proper electrodeposition of platinum at surface sites.

The remarkable clearcut and flat step structures which are observed in the STM images for the three types of preferred oriented platinum can be understood through the electrochemical mechanism described above by taking into account the combined effect of the electrodissolution of platinum mainly at corners, that is, at sites where metal atoms are most loosely bound to the lattice, and the electrodeposition process undergoing preferentially at kink sites. The overall effect leads to an increase in the length of the steps and the development of extended terraces.

In summary, we report on a measurement of the surface topography of electrochemically faceted electrodes by STM. The data show features characteristic of the development of well-defined orientations and constitute an 
attempt to correlate microscopic observations at the nanometer scale with macroscopic voltammetric data.

\section{Acknowledgements}

Financial support was obtained from CAICyT under contracts 14226/82 and 0386/84, from Fundación Ramón Areces and Centro Científico UAMIBM.

\section{References}

[1] R.M. Cerviño, W.E. Triaca and A.J. Arvía, J. Electrochem. Soc. 132 (1985) 266.

[2] J.C. Canullo, W.E. Triaca and A.J. Arvia, J. Electroanal Chem. 175 (1984) 337.

[3] J.C. Canullo, W.E. Triaca and A.J. Arvia, J. Electroanal. Chem. 200 (1986) 397.

[4] R.M. Cerviño, A.J. Arvía and W.E. Vielstich, Surface Sci. 154 (1985) 623.

[5] D.M. Kolb, J. Vacuum Sci. Technol. A4 (1986) 1294.

[6] J. Gómez, L. Vázquez, A.M. Baró, N. Garcia, C.L. Perdriel, W.E. Triaca and A.J. Arvia, Nature, 323 (1986) 612.

[7] L. Vázquez, J. Gómez, A.M. Baró, N. Garcia, M.L. Marcos, J. González Velasco, J.M. Vara, A.J. Arvia, J. Presa, A. García and M. Aguilar, J. Am. Chem. Soc. (1987), in press.

[8] G. Binnig and H. Rohrer, Sci. Am. 253 (1985) 50.

[9] C.L. Perdriel, W.E. Triaca and A.J. Arvía, J. Electroanal. Chem. 205 (1986) 279.

[10] N. Ibl. Surface Technol. 10 (1980) 81.

[11] See for example, J.V. Sanders, in: Chemisorption Reactions on Metallic Films, Ed. J.R. Anderson (Academic Press, London, 1971) pp. 1-38. 